\title{
Synchronization of Suspension Plasma Spray Injection with the Arc Fluctuations
}

\author{
J. Krowka, V. Rat, S. Goutier, and J.F. Coudert
}

(Submitted December 20, 2013; in revised form February 6, 2014)

\begin{abstract}
Poorly controlled heat and momentum transfers between plasma and material, plasma instabilities are some of the difficulties encountered in suspension plasma spraying. The improvement of this method is usually attempted by means of the reduction of arc fluctuations. This paper presents a new approach to the injection of reactive material in an arc jet. The principle is to produce a pulsed laminar plasma jet combined with phased injection of liquid droplets. This is achieved by the particular design of the plasma torch that works at moderate power and following a resonant mode. The droplets are injected using a piezoelectric device, based on drop-on-demand method, triggered by the voltage signal sampled at the torch connections. The results are evaluated by time-resolved imaging technique that shows how the trajectories are influenced by the moment at which the droplets penetrate the plasma jet.
\end{abstract}

Keywords drop-on-demand method, heat and momentum transfer, piezoelectric injection, pulsed arc plasma, suspension plasma spraying

\section{Introduction}

For several years, in the field of coatings technology, the suspension plasma spraying has been a well-established process which allows obtaining the finely structured and dense coatings. They can be used in wear-resistant applications as well as in more complex integrated devices, such as solid oxide fuel cells, photocatalytic coatings. However, in more complex applications, the researches face many problems, such as obtaining the appropriate crystallographic structure of materials, e.g., perovskite structure of cathode in SOFC or anatase phase in $\mathrm{TiO}_{2}$ coatings (Ref 1,2). As Henne (Ref 3 ) has presented on the example of the SOFC cathode, these more complex devices can be produced by other thermal spray methods, such as vacuum plasma spraying (VPS). However, the goal of technological development is the reduction of costs which can be achieved by suspension plasma spraying using DC torches at atmospheric pressure. Consequently, the special efforts have been made to improve the suspension plasma spraying method. In recent years, the research has been focused on the improvement of heat and momentum transfers between material and plasma produced by the plasma torch that is usually attempted by means of the reduction of arc instabilities. The analysis of the operating conditions performed by Duan and Heberlein (Ref 4) and Rat and Coudert (Ref 5) shows that the

J. Krowka, V. Rat, S. Goutier, and J.F. Coudert, CNRS University of Limoges, SPCTS UMR7315, European Ceramic Centre, 12 rue Atlantis, 87068 Limoges Cedex, France. Contact e-mail: joanna.krowka@unilim.fr.

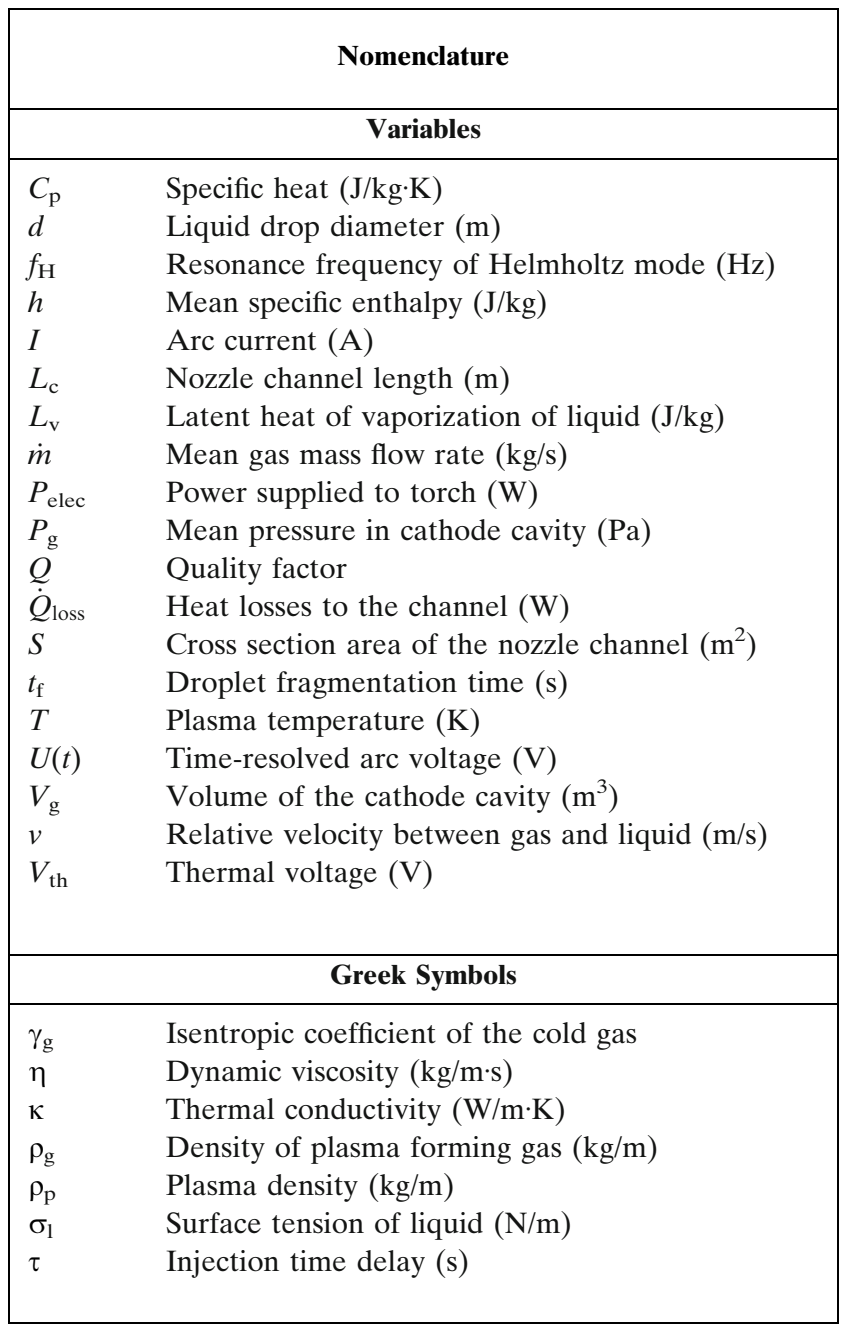

parameters such as arc current, anode erosion, gas flow rate, and composition of plasma forming gases are clearly correlated with the fluctuations of plasma jet. However, 
the adjustment of these operating conditions decreases the arc voltage variations in a negligible way. One of the solutions to reduce these instabilities was presented in Ref 6 . The additional acoustic resonator mounted on the torch led to modify the pressure inside the cathode cavity. It resulted in significant reduction of the arc voltage fluctuations. The other solution is to develop new non-conventional plasma torch. One of the examples is PJ 100, invented by Plasma Jet Co., with a fixed minimal arc length. Vilotijevic et al. (Ref 7,8) have shown that plasma produced by this PJ 100 gun has better flow laminarity, temperature homogeneity, and higher velocity than the jet generated by the conventional plasma torches. Another approach to plasma torches is the torch system characterized by three parallel cathodes. This special design can be found in TRIPLEX (Sultzer-Metco, Switzerland) or Mettech Axial III (Northwest Mettech Corp., North Vancouver, BC, Canada) (Ref 9, 10). This characteristic construction results in the elongation of the arcs which leads to higher arc voltage and enthalpy of the plasma jet. The arc root fluctuations are reduced and the treatment of the injected material is more homogeneous. However, the studies (Ref 11) show that despite the elongation of the arcs and consequently reducing significantly the voltage fluctuations, the plasma produced by these torches still remain non-uniform. Therefore, this paper highlights a new approach to arc fluctuations by the increase in a controlled way the instabilities associated with a synchronous injection of materials. To synchronize the suspension injection, the method capable to control the moment of material injection is required. In the conventional systems the injection is carried out by the atomization and mechanical injection. The first method uses the atomizing gas to break up the liquid into droplets (Ref 12). Fazilleau et al. (Ref 13) have noticed that the atomizing gas flow rate is the important parameter in this method and its control can limit the dispersion of droplets trajectories. In the second method, mechanical injection, the suspension is injected through the stainless steel tube by controlling the pressure of the liquid in the reservoir (Ref 13). In these conventional injection systems the size and velocity of suspension droplets can be slightly dispersed. Alternatively, Blazdell and Kuroda (Ref 14) have highlighted the possibility of suspension injection by superimposing a modulation signal to piezoceramic material of dc jet printer head. The liquid has been forced through the nozzle under pressure, superimposed by a piezoelectric drive rod modulated with frequency of $64 \mathrm{kHz}$. This method allows obtaining the continuous injection of individual droplets of $100 \mu \mathrm{m}$. It permits control of the droplet diameter, flow rate, and velocity. However, the suspension is injected continuously to the plasma jet. In the presented work the system capable to control the moment of material injection has been used. This requirement is met by the injection technique based on drop-on-demand (DOD) method commonly applied in the inkjet printing (Ceradrop Company, Limoges, France).

The purpose of this paper focuses on obtaining the resonant arc plasma source associated with a synchronous material injection. First of all, the plasma instabilities in dc torch and the methods allowing decreasing these fluctuations have been examined and presented above. These studies have resulted in the coupling of Helmholtz and restrike oscillations in a new resonant mode which has allowed achieving the pulsed laminar plasma jet. It is expected that this pulsed jet synchronized with a suspension injection can be able to better control of heat and momentum transfers between plasma and materials. The next parts, 2, 3, and 4, show the results of this work, pulsed laminar plasma jet combined with phased injection of liquid droplets. The section 5 gives the conclusions.

\section{Experimental}

\subsection{Mosquitorch}

In plasma spraying method, the plasma jet is produced by the torch, in this work, supplied by direct current source, presented in Fig. 1. The cathode tip ignites the gas, fed to the torch (gas input in Fig. 1), to form the plasma, which flows through the extended anode nozzle and emerges out at a high velocity through the exit of the torch. The previous studies (Ref 5, 15, 16) demonstrated that plasma torch, even powered by dc regulated source, generates highly fluctuated plasma jet. The "stick and slip" motion of the arc at the nozzle wall is one of the sources of these fluctuations, the so-called restrike mode. This kind of relaxation oscillation is due to the elongation of electrical current paths of the arc by the gas flow, followed by a backward rearcing which is initiated by the electrical breakdown of the cold gas layer that isolates the arc column from the nozzle wall. Restrike mode gives a voltage signal which is approximately saw tooth in shape. The other major source of the plasma fluctuations has been recently identified in plasma torches (Ref 15, 16). These instabilities appear in power spectrum of the arc voltage as a strong, sharp peak $(2-5 \mathrm{kHz})$ which proves the presence of a resonant phenomenon inside the plasma torch. It has been demonstrated that this main fluctuation is due to compressibility effects of plasma forming gas in the cathode cavity and it is referred to Helmholtz mode. The resonance frequency, $f_{\mathrm{H}}$, linked to this oscillation can be rather accurately predicted as a function of the electrode configuration and the operating parameters, according to the following equation:

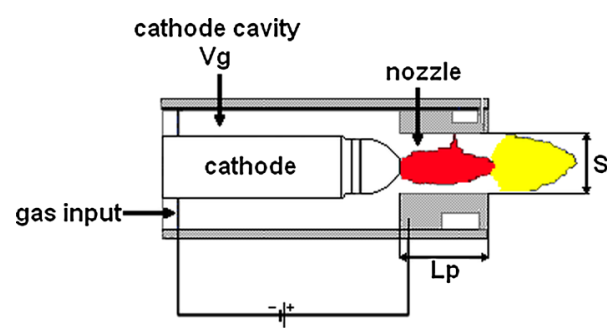

Fig. 1 Scheme of a direct current plasma torch (1) 
$f_{\mathrm{H}}=\frac{1}{2 \pi} \sqrt{\frac{\gamma_{\mathrm{g}} p_{\mathrm{g}}}{\rho_{\mathrm{g}}} \frac{S}{L_{\mathrm{c}} V_{\mathrm{g}}}}$,

where $\gamma_{\mathrm{g}}$ and $P_{\mathrm{g}}$ are, respectively, the isentropic coefficient of the cold gas and the mean pressure in cathode cavity; $\rho_{\mathrm{p}}$ is the plasma density; $S, L_{\mathrm{c}}$, and $V_{\mathrm{g}}$ are, successively, the cross section area, the length of the nozzle channel, and the volume of the cathode cavity, as shown in Fig. 1.

We present the possibility of coupling Helmholtz and restrike modes of instabilities to obtain pulsed laminar arc plasma jet (Ref 17,18$)$. In this case, the arc root at the nozzle wall experiences a very regular back and forth movement with highly repeatable rearcing leading to the emission of thermal plasma balls at the resonance frequency. The coupling of pressure oscillations and rearcing phenomena is carried out in a special torch characterized by a rather large cathode cavity $\left(V_{\mathrm{g}}=17.8 \mathrm{~cm}^{3}\right)$ to reinforce the $Q$ factor, to decrease the specific frequency of the Helmholtz mode and also by a short length so that the longitudinal acoustic modes are rejected to a high frequency region. This torch works at a moderate power, with regulated arc currents between 8 and $35 \mathrm{~A}$. Nitrogen is used as plasma gas with mean mass flow rates, $\overline{\dot{m}}$, between 0.042 and $0.104 \mathrm{~g} / \mathrm{s}$ and different nozzles are tested with channel diameters, $d$, of $2.5,3,3.5$, and $4 \mathrm{~mm}$ and a single channel length, $L_{\mathrm{c}}=13 \mathrm{~mm}$. Figure 2 depicts the arc voltage recorded in this specific mode, when restrike and Helmholtz modes are locked together. The time-resolved measurement of the arc voltage, $U(t)$, is performed using a data acquisition computer card piloted by the LabView software. The arc voltage signal, reduced by a voltage divider, is sampled at $320 \mathrm{kS} / \mathrm{s}$ rate during $0.2 \mathrm{~s}$. A very regular voltage signal, presented in Fig. 2, is the result of adjusting the operating parameters, such as mean mass flow rate, the channel diameter, and the arc current, described in details in Ref 17 and 18. This resonant mode $(\sim 700 \mu$ s period), called mosquito mode because of the sound produced by a torch that recalled the flight of a mosquito, is obtained for $d=4 \mathrm{~mm}, \overline{\dot{m}}=0.042 \mathrm{~g} / \mathrm{s}$ and $I=14.9$ A. Then, this special torch will be called "mosquitorch" in the following paper.

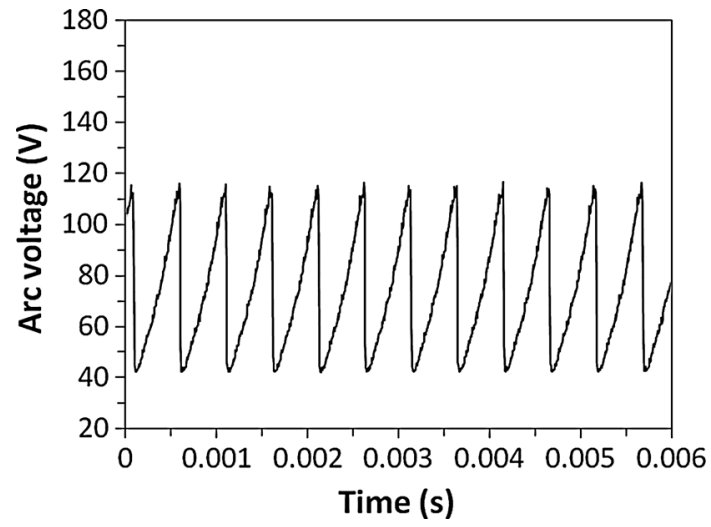

Fig. 2 Arc voltage signal for: $d=4 \mathrm{~mm}, I=14.9 \mathrm{~A}, \mathrm{~N}_{2}: 0.042 \mathrm{~g} / \mathrm{s}$, $\bar{V}=74 \mathrm{~V}$
For each experiment the heat losses, $Q_{\text {loss }}$, to the electrodes are measured together with the electric power supplied to the torch, $P_{\text {elec}}$. The mean specific enthalpy of the plasma is then deduced following the relationship:

$h=\frac{\left(P_{\text {elec }}-Q_{\text {loss }}\right)}{\overline{\dot{m}}}$

The energy balance measurements reveal that heat losses per unit time in the cooling circuits of electrodes are proportional to the arc current, so that an equivalent "thermal" voltage, $V_{\mathrm{th}}$, can be defined with $Q_{\text {loss }}=I \cdot V_{\mathrm{th}}$. In the case presented in Fig. $2, V_{\mathrm{th}}=36 \mathrm{~V}$ and the mean value for the torch voltage is $\bar{V}=74 \mathrm{~V}$. The efficient voltage, which is useful for the conversion of electric power into enthalpy, is then the difference $V-V_{\text {th }}$ that changes from 6 to $80 \mathrm{~V}$ within $700 \mu \mathrm{s}$. As a consequence, the power supplied to the gas is modulated in the range 90-1200 W. The specific enthalpy is modulated in the same proportions, $h_{\max } / h_{\min }=13$, with a mean value of $13.3 \mathrm{MJ} / \mathrm{kg}$. The plasma jet is observed by means of a camera triggered (PCO, Kelheim, Germany) at a given moment in one period of voltage cycle $(\sim 700 \mu \mathrm{s})$, obtained by different trigger signals, as presented in Fig. 3. The camera aperture time is $60 \mu \mathrm{s}$, and 100 shots are recorded in both situations (a) corresponding to a trigger $210 \mu \mathrm{s}$ and (b) to a trigger $520 \mu$ s after a falling front of the voltage.

Figure 3 proves the strong modulation of the plasma in mosquito mode. Figure 3(a) presents the plasma pulse characterized by a lower enthalpy compared to Fig. 3(b). The examination of 100 sequences for each run showed also a very regular evolution of the plasma in each cycle.

\subsection{Experimental Setup}

The suspension spraying system, used in the experiment, consists of the new home-made torch described
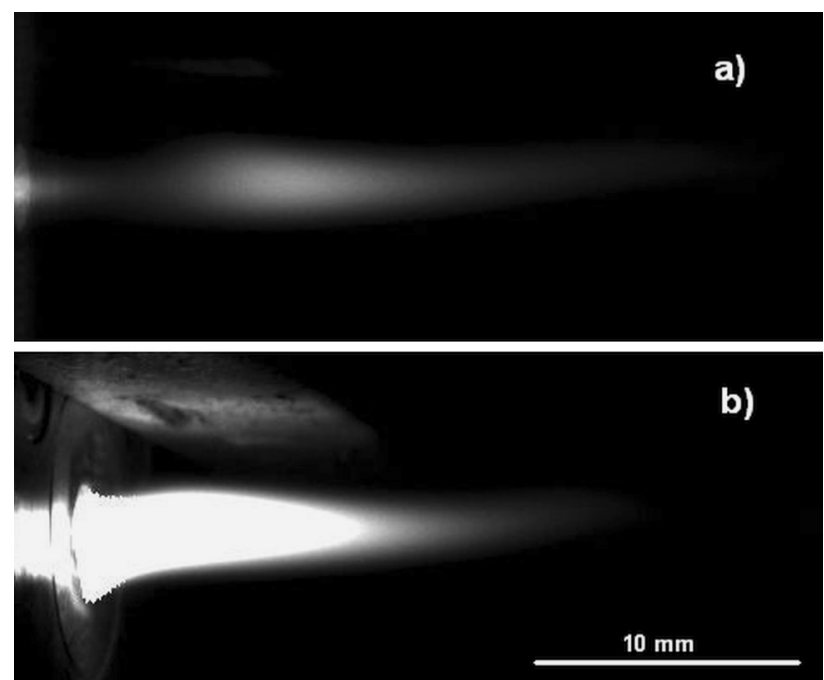

Fig. 3 Time-resolved imaging (aperture time $60 \mu \mathrm{s}$ ) of pulsed arc jets for different trigger time delays after voltage falling (see Fig. 2) front in one period: (a) $210 \mu \mathrm{s}$ and (b) $520 \mu \mathrm{s}$ 


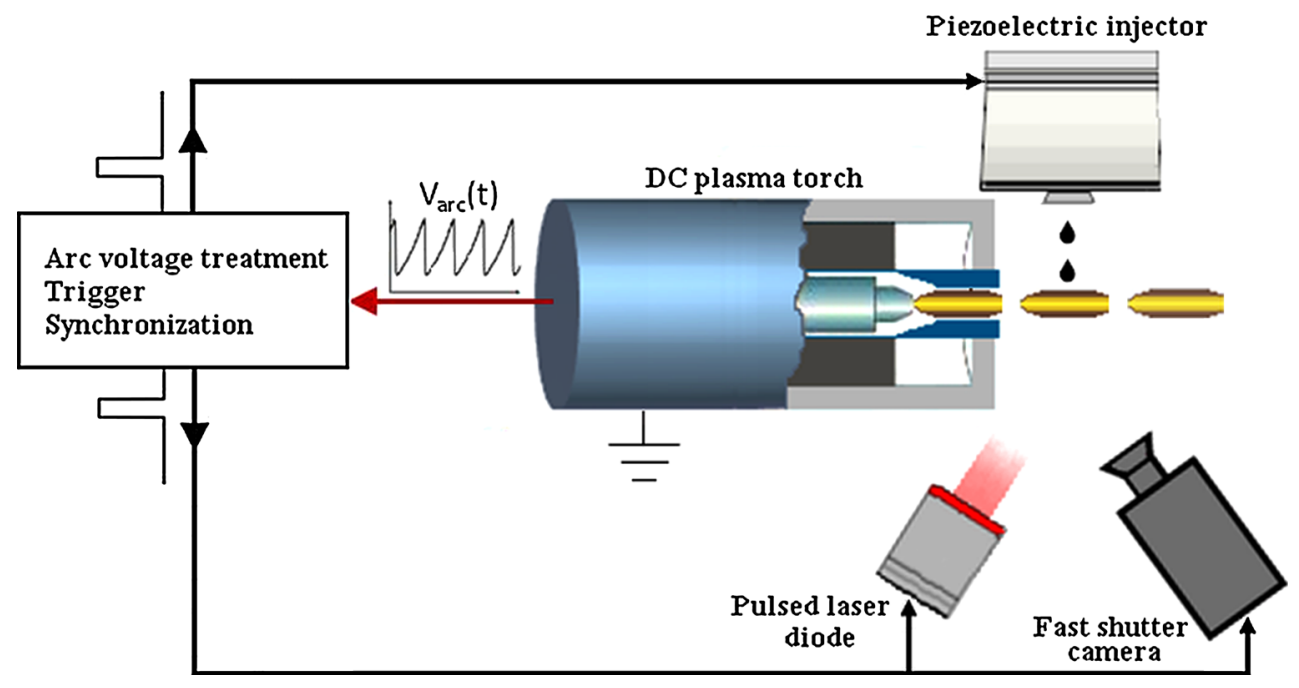

Fig. 4 Schematic view of experimental setup including pulsed arc plasma torch, time-resolved imaging, piezoelectric injector, and synchronization treatments

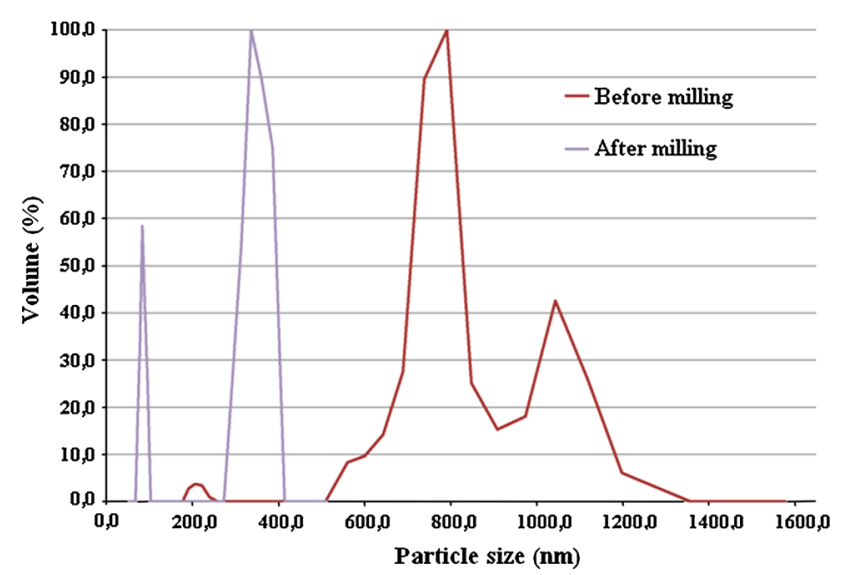

Fig. 5 The particle size distribution of $\mathrm{TiO}_{2}$ measured before and after milling

above, equipped with an external injector based on the DOD method, as shown in Fig. 4.

The aqueous suspension is stored within a buffer tank and is composed of $\mathrm{TiO}_{2}$ crystallites (42 wt.\%) with mean diameter of $200 \mathrm{~nm}$ (Ref 19). Figure 5 presents the particle size distribution of titanium dioxide $\left(90 \% \mathrm{TiO}_{2}\right.$ rutile phase) powder used to formulate the suspension in the experiment. Before the milling process the particle size distribution varies from $200 \mathrm{~nm}$ to $1.3 \mu \mathrm{m}$, after milling the particle size is reduced and is ranging from 100 to $350 \mathrm{~nm}$.

The droplets are injected into the plasma jet using a piezoelectric head actuated by voltage pulses carefully shaped to avoid satellite droplets and to adjust the velocity of single calibrated droplets $(50 \mu \mathrm{m}$ in diameter $)$ between 2 and $10 \mathrm{~m} / \mathrm{s}$. The pulse shape must also be adapted to the rheological properties of the suspension. The piezoelectric injector can be triggered at frequencies up to $20 \mathrm{kHz}$ and comprises a ramp of 128 individual micro-nozzles, disposed perpendicular to the plasma jet axis, with a step of

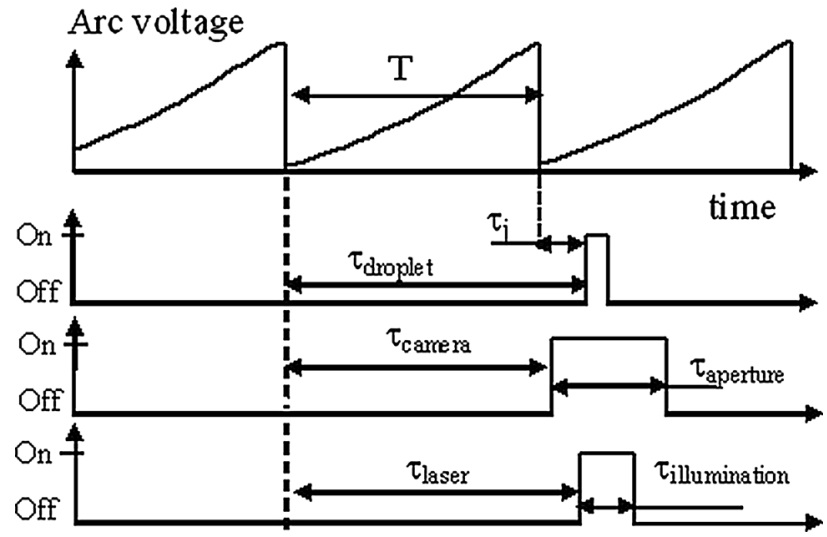

Fig. 6 Timing for droplet emission and synchronous imaging. $T$, $\tau_{\text {camera }}$, and $\tau_{\text {laser }}$, are, respectively, the arc voltage period, the internal delay time of camera, and the delay time of laser. $\tau_{\text {aperture }}$ is an adjustable time at which camera aperture is opened. $\tau_{\text {illumination }}$ is $1 \mu$ s laser pulse illumination. $\tau_{j}$ is the time at which a droplet penetrates the plasma after a falling front of the voltage and is chosen by adjusting $\tau_{\text {droplet }}=n T+\tau_{j}$

$0.5 \mathrm{~mm}$. A single nozzle, located in the middle of the ramp, was used for that work. The injection system is protected from heat flux coming from the plasma by a water-cooled copper screen (not shown in Fig. 4) in which a horizontal slit, $10 \mathrm{~mm}$ long and $1 \mathrm{~mm}$ wide, is machined. The droplets pass through that slit and travel at least a distance of $5 \mathrm{~mm}$ between the piezoelectric head and the plasma jet axis, giving rise to a time of flight that must be accounted for in the timing process. The emission of each droplet, based on DOD method, is triggered from sampling of the torch voltage after an adjustable delay, $\tau_{\text {droplet }}$, counted after a falling front (Fig. 6), so that the suspension penetrates the plasma jet as a train of individual droplets. Following the mosquito mode, the plasma ball emissions and the droplet ejections constitute two series of periodic events of same period whose mutual phase can be controlled by $\tau_{\text {droplet }}$. 


\subsection{Fast and Time-Resolved Imaging System}

The interaction between the plasma and the droplets is first observed using a high-speed camera with 6000 frames per second at $1024 \times 144$ pixels resolution $(\mathrm{PCO}$, Kelheim, Germany). This allows following a single plasma ball during its own flight, but the imbedded droplet cannot be seen in the same time because of their very low luminosity compared with that of the plasma. A second imaging system has been set up (Fig. 4), which is able to see simultaneously the droplets, the plasma, and also the solid particle heated up after solvent vaporization. It is composed of the fast shutter camera $(1392 \times 1040$ pixels $)$ already used for Fig. 3, and a pulsed laser diode (HiWatch, Oseir, Tampere, Finland, wavelength: $801 \mathrm{~nm}$, peak power: $50 \mathrm{~W}$ ). The laser is providing a sheet of light thanks to a specific optical system. During that application, the camera is equipped with an interferential filter $(\Delta \lambda=50 \mathrm{~nm})$ to reduce plasma radiation. Both the camera and the laser are triggered following a procedure which is sketched in Fig. 6 and it differs slightly from that used in Fig. 3 . The different delays, $\tau_{\text {droplet }}, \tau_{\text {camera }}$, and $\tau_{\text {laser }}$ can be chosen independently, but after the same falling front of the torch voltage.

\section{Results}

Figure 7 displays four successive pictures (6000 frame/ sec), about $166 \mu$ s between each, taken with the highspeed camera with an exposure time of $100 \mu \mathrm{s} . \tau_{\text {droplet }}$ is adjusted so that each droplet reaches the plasma axis $\tau_{j}=$ $260 \mu$ s after a voltage falling front. Compared to that of Fig. 3, the observation area is shifted by approximately $1 \mathrm{~cm}$ downstream of the torch exit. On the left of Fig. 7(a), the tip of the arc jet is presented that contains no droplet. The spherical shape that follows immediately on the right is the result of a droplet injected in the preceding period. What is seen is the result of the interaction between the
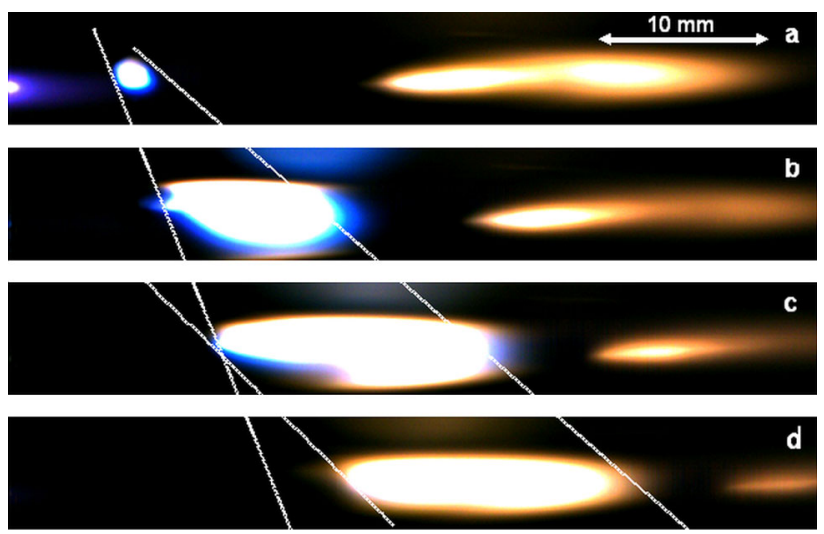

Fig. 7 Fast imaging (6000 frames/s, time aperture: $100 \mu \mathrm{s}$ ) of synchronized injection of $\mathrm{TiO}_{2}$ suspension in nitrogen pulsed arc plasma jet for the injection time delay $\tau_{j}=260 \mu \mathrm{s}$. Time is increased by $166 \mu$ s between successive pictures (a), (b), (c), and (d) (enhanced on-line) plasma and the solvent at the beginning of the vaporization process? The right part of the picture shows the result of the interaction with a droplet injected two periods before. Figure 7(b) follows Fig. 7(a) by $166 \mu$ s. The spherical shape has been strongly expanded and lengthened, but the sharp details at the left of the plasma ball suggest that a certain amount of liquid still remains non-vaporized. The expansion is continued in Fig. 7(c) but seems to reach a maximum in Fig. 7(d). The dotted lines drawn on this figure show that the front part of the plasma ball travels at a speed approximately twice that of the rear part, which is consistent with the expansion process. The rear part lags behind the plasma ball because it still contains a small amount of suspension under acceleration. Once the vaporization is complete, the plasma ball travels as a whole as it can be seen in Fig. 7(c) and (d). A picture taken $166 \mu$ s after the situation of Fig. 7(d) should be similar to Fig. 7(a). This can be checked on the associated video that testifies of the reproducibility of the process. It must be underlined that the brightness is drastically increased as soon as a droplet penetrates the arc jet. The amount of nitrogen that flows during one period, that is $T=700 \mu$ s, is approximately $310^{-8} \mathrm{~kg}$ and the energy content in one plasma ball is $0.5 \mathrm{~J}$. The mass of a droplet entering one of them is $6 \times 10^{-11} \mathrm{~kg}$ and the energy available is high enough to vaporize completely the solvent and liberate solid particles. The plasma is then seeded with new species that radiate intensively. This light emission requires spectroscopic investigation and cannot be interpreted at the step of this work. However, some qualitative features can be deduced from comparative observations.

Starting from the expansion of a droplet as shown in Fig. 7(a), the evolution of successive volumes over a sequence of four pictures is measured by image analysis. It is supposed that the resulting plasma balls have an ellipsoidal shape, whose volume is $V=(4 \pi / 3) \Delta x \Delta y \Delta z$, i.e., assuming that the radial expansion is isotropic $(\Delta x \sim \Delta y)$ and $z$ being the axial coordinate. Figure 8 presents

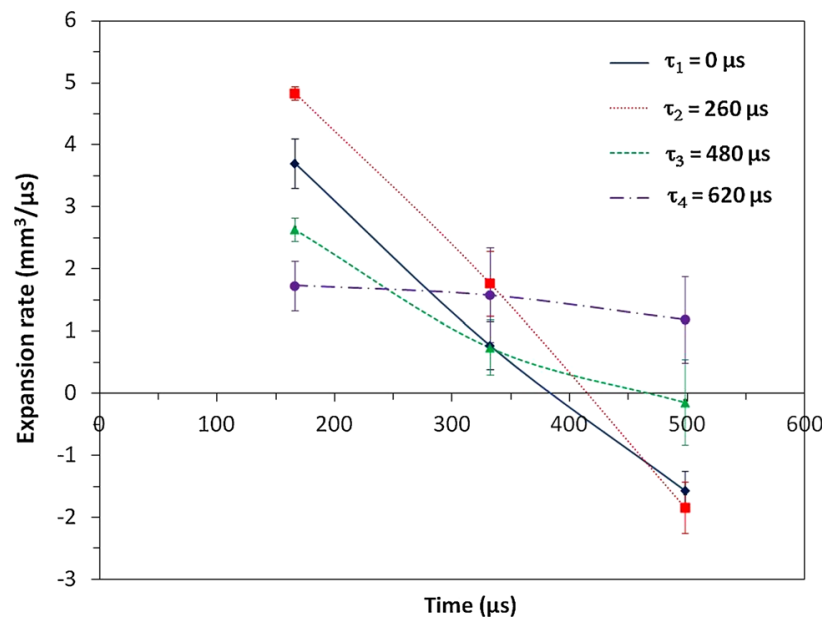

Fig. 8 Dependence of measured expansion rates of plasma balls on the injection time delay $\tau_{j}$ that are defined in Fig. 7 

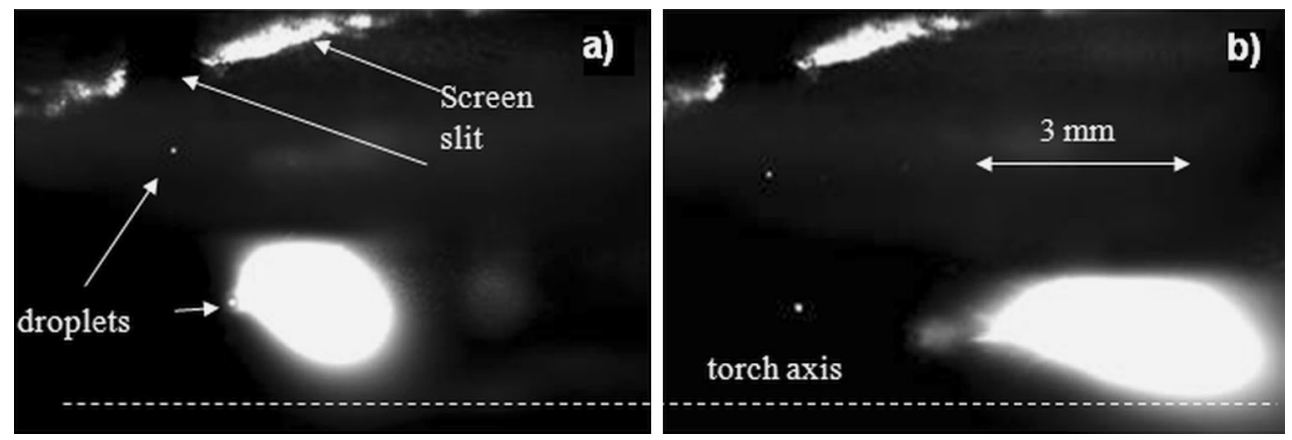

Fig. 9 Influence of the local instantaneous specific enthalpy on droplet thermal treatment. For the lower droplet, (a) high level of local specific enthalpy, (b) low level. Camera exposure: $5 \mu$ s. Laser illumination: $1 \mu \mathrm{s}$

measurements of expansion rates of droplets during plasma treatment for the different injection time delays, $\tau_{j}$. The expansion rate is $\left(V_{i}-V_{i-1}\right) / \Delta t$ where $V_{i}$ is the volume at the instant $t_{i}=i \times \Delta t, \Delta t=166 \mu \mathrm{s}, i \in[0 ; 3]$ and where $t_{0}$ corresponds to Fig. 7(a). The standard deviation results from the measurements of volumes for 10 similar sequences of pictures. Figure 8 shows that the expansion rate substantially depends on $\tau_{j}$, with $j=1 \ldots 4$, especially during the first $166 \mu$ s. Later, the expansion rate decreases and becomes negative for $\tau_{1}=0 \mu \mathrm{s}$ and $\tau_{2}=260 \mu \mathrm{s}$, meaning that plasma starts to recombine. This dependence on $\tau_{j}$ was expected because as shown in Ref 17 the net specific enthalpy available in the plasma is strongly modulated in the proportion, $h_{\max } / h_{\min } \approx 13$, with a mean value: $\bar{h}=13.3 \mathrm{MJ} / \mathrm{kg}$. Consequently, heat transfers to droplets are also affected by this modulation and the expansion rates as well.

The influence of the time at which a droplet penetrates the pulsed arc jet has been investigated using the second optical system, what is able to visualize both the plasma ball and the droplets thanks to the laser illumination. The triggered camera is equipped with different objectives that allow the observation with different magnifications. In Fig. 9 the camera and the laser are delayed by the same time after the droplet emission, but the droplets penetrate the plasma earlier in Fig. 9(a) than in Fig. 9(b), that is obtained by changing $\tau_{j}$. The "pure" nitrogen plasma at the left of the pictures cannot be seen because its luminosity is too low compared with that of the seeded plasma ball or that of the laser illuminated droplets. The exposure time for the camera is $5 \mu$ s for both pictures. In Fig. 9(a), the lower droplet penetrates the plasma at a moment corresponding to a situation presented in Fig. 3(b). At that time the local specific enthalpy is high and the vaporization-seeding process immediately begins.

In Fig. 9(b) the lower droplet enters the plasma with a low level of local specific enthalpy, a situation similar to the one of Fig. 3(a). At that time, the vaporization-seeding process does not concern this droplet but the injected one period earlier and it gives the plasma ball at the right of Fig. 9(b). Figure 10(a)-(d) present time-resolved imaging of the dynamic interaction between the plasma jet and the droplets. These pictures are obtained with a low-magnification objective for the different time delays over one
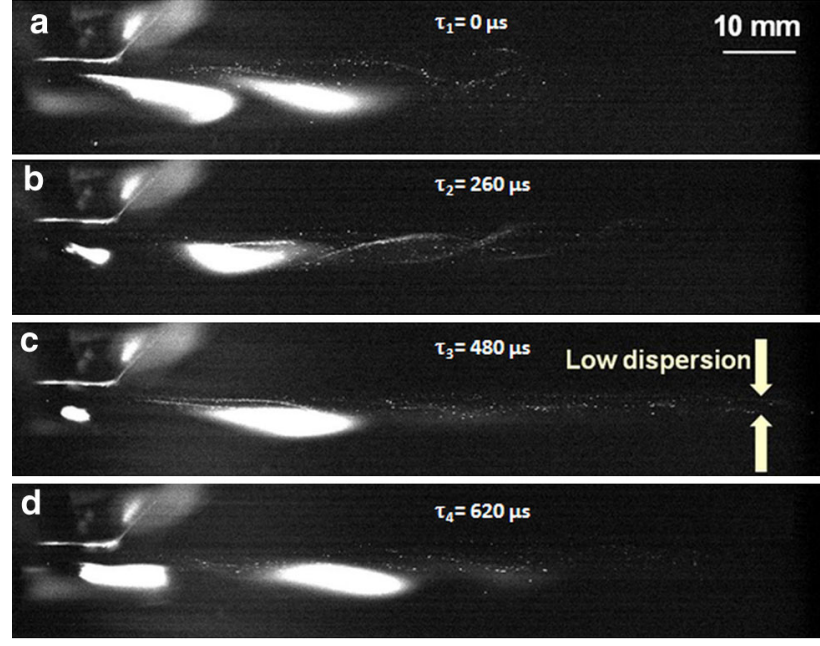

Fig. 10 Time-resolved imaging of synchronized injection of ceramic $\mathrm{TiO}_{2}$ suspensions with laser illumination for different injection time delay $\tau_{j}$

period. The aperture time is $10 \mu \mathrm{s}$ for the camera and the laser pulse duration for illumination is $1 \mu \mathrm{s}$. It is chosen so that the distance traveled by droplets or materials is small compared to the picture resolution to ensure that the observed phenomena are indeed frozen. The laser shot allows visualizing the solid particles left in the jet after solvent vaporization, but their individual images are strongly oversized by diffraction, scattering, or resolution due to pixel size. The velocities of the center of mass of plasma balls can also be measured by image analysis and vary between 30 and $50 \mathrm{~m} / \mathrm{s}( \pm 10 \mathrm{~m} / \mathrm{s})$ depending on the injection delay $\tau_{j}, j=1-4$, as shown in Fig. 10. Several hundreds of pictures similar to those of Fig. 10 were recorded and they display the same features provided they are triggered with the same $\tau_{j}$. These pictures clearly demonstrate that the trajectories and related thermal history of injected materials depend on the moment when droplets penetrate into the plasma. In case of $\tau_{1}=0 \mu \mathrm{s}$, the most significant part of materials travels in the plasma fringes giving rise to large dispersion of trajectories $(\sim 10 \mathrm{~mm})$ and limited axial distance of material transport $(\sim 40-60 \mathrm{~mm})$. In case of $\tau_{2}=260 \mu \mathrm{s}$, the entrainment of 


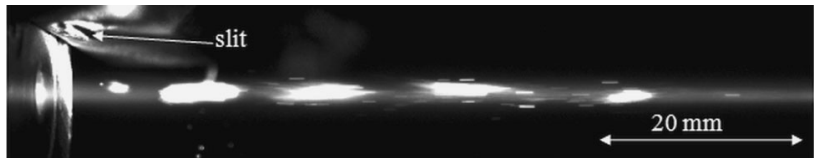

Fig. 11 Visualization at low magnification. Camera time aperture: $60 \mu \mathrm{s}$, laser illumination: $1 \mu \mathrm{s}$, no interferential filter

materials in the plasma core is improved with a lower radial dispersion and a slightly increased transport distance. It is also interesting to observe alternate and intricate trajectories on this second picture, meaning that the pulsed plasma jet is trapping particles all along its own axis. This effect seems to be due to the pulsed emission of the plasma balls that alternate their curvatures by interacting between each others. This gives a certain spatial coherence for the plasma jet and also recalls some visual effects such as the Von Karman Street in vortices emission. Moreover, this phenomenon requires more studies and it is the subject of future research. In case of $\tau_{3}=480 \mu \mathrm{s}$, a low material dispersion is observed corresponding to the longest transport distance. At last, the case of $\tau_{4}=620 \mu \mathrm{s}$, because it is almost the $700 \mu \mathrm{s}$ period, resembles the first case $\tau_{1}=0$.

The picture (Fig. 11) is recorded at low magnification to increase the observation area and with a time aperture of the camera of $60 \mu \mathrm{s}$. The interferential filter is removed so that the picture integrates the traces of incandescent solid particle whose speed is estimated in the range 30$40 \mathrm{~m} / \mathrm{s}$. The light reflection on the front part of the torch and on the thermal screen equipped with the slit is due to the laser shot $(1 \mu \mathrm{s})$. The plasma is in a situation intermediate between those of Fig. 3(a) and (b) and the droplet that can be seen on the picture is imbedded in the preceding plasma ball and begins to vaporize. Five seeded plasma balls can be distinguished that highlight the long distance coherence of the plasma jet.

\section{Discussion}

The thermo-physical phenomena which occurred at the droplet scale have been analyzed by models presented by Fazilleau et al. (Ref 13) and Basu et al. (Ref 20). The first model given by Fazilleau focuses on the suspension droplet. Basu et al. have highlighted the phenomena associated with solution precursor plasma spray (SPPS) process. Once the droplet is entrained into plasma jet, it undergoes the fragmentation process due to a strong shear stress. Fazilleau has showed that the fragmentation time is given by:

$t_{\mathrm{f}}=\frac{8 \sigma_{1}\left(d_{1} / d_{\min }-1\right)}{C_{\mathrm{D}} \rho_{\mathrm{p}} v^{3}}$,

where $\sigma_{1}, C_{D}, \rho_{p}$, and $v$ are, respectively, the surface tension of liquid, the plasma drag coefficient, plasma density, and the velocity between plasma and liquid drop. The initial droplet diameter $d_{1}$ is fragmented into $n$ droplets of $d_{\text {min }}$ defined as $d_{\min }=8 \sigma_{1} / C_{\mathrm{D}} \rho v^{2}$. The time associated with this process is of the order of microseconds and significantly shorter than the vaporization time which has been showed in Ref 13. The fragmentation process depends notably on the Weber number given by: $W e=$ $\rho_{\mathrm{g}} \cdot v^{2} \cdot d / \sigma_{1}$, where $\rho_{\mathrm{g}}, v, d$, and $\sigma_{1}$ are, respectively, the gas mass density, the relative velocity between the gas and the liquid, the diameter of the droplet, and the surface tension of the liquid. Saha et al. (Ref 21) and Basu et al. (Ref 20) have shown that a Weber number of about 14 has been the critical value over which the droplet undergoes breakup. In the present experimental conditions where the mean specific enthalpy is $13.3 \mathrm{MJ} / \mathrm{kg}$, the Weber number ranges between 0.2 and 0.3 . The plasma thermodynamic and transport properties are given by tables found in Boulos et al. (Ref 22). Consequently, fragmentation processes should not occur because the Weber number is much smaller than the critical value determined by Saha et al. and Basu et al. Figures 7(a) and 9(a) indeed present the droplet treatment in which the droplet fragmentation process seems not to be observed. The second phase of the droplet treatment in the plasma is heating and vaporization. All the droplets introduced into the plasma jet undergo heating resulting in solvent vaporization which is obtained by the energy balance equations:

$\frac{d\left(d_{\mathrm{m}}\right)}{d t}=-\frac{Q}{\pi d^{2} \rho_{\mathrm{p}} L_{\mathrm{V}}^{\mathrm{l}}}$,

where $\rho_{\mathrm{p}}, L_{\mathrm{V}}$, and $d$ are, respectively, the plasma density, the latent heat of vaporization of liquid, and the droplet diameter. $Q$ is the plasma heat transferred by conduction/ convection to the particle from the plasma jet, equals to:

$Q=4 \pi r^{2} \frac{\kappa}{C_{\mathrm{p}}} \frac{d h}{d r}$,

where $\kappa$ is the thermal conductivity, $C_{\mathrm{p}}$ the specific heat, and $h$ the specific enthalpy of the plasma (Ref 23). As it has been presented above, the arc voltage recorded in the mosquito mode is characterized by a very regular sawtooth-shaped waveform. The enthalpy, measured in these conditions, is modulated with a ratio $h_{\max } / h_{\min }=13$, with a mean value of $13.3 \mathrm{MJ} / \mathrm{kg}$. While considering the plasma heat equation, it can be stated that the heat transferred to the particle from the plasma jet is also modulated which will be investigated in the next step of this work. The vaporization time has been deduced by:

$t_{\mathrm{V}}=\frac{L_{\mathrm{V}} \rho r^{2}}{\left(T-T_{1}\right) \kappa N u}$,

where $L_{\mathrm{V}}, r, T, T_{1}$, and $\kappa$ are, respectively, the latent heat of vaporization of liquid, the droplet radius, the plasma temperature, the temperature of the droplet, and the thermal conductivity. $N u$ is the Nusselt number defined by the tables (Ref 22) and equals to 2.6. The vaporization time calculated for the experimental conditions is equal to $25 \mu \mathrm{s}$.

The acceleration time, also known as Stokes time, has been determined by the equation presented in Ref 24: 
$t_{\mathrm{a}}=\frac{\rho d^{2}}{18 \mu_{\mathrm{c}}}$,

where $d$ and $\mu_{\mathrm{c}}$ are, respectively, the particle diameter and the gas dynamic viscosity. Under the experimental conditions it has been noticed that the droplet acceleration time is equal to around $800 \mu \mathrm{s}$ and is much greater than the vaporization time which can be observed in Fig. 7.

This means that vaporization occurs much faster than the acceleration produced by the plasma flow. Note that in conventional suspension plasma spraying with a mechanical injection of suspensions, fragmentation processes occur before vaporization of droplets. In the case of synchronized injection of suspensions in pulsed arc, the plasma treatment is completely ruled by heat transfers.

Fauchais et al. have highlighted that the treatment of particles within the droplet, after the vaporization of the solvent, depends on their size distribution, morphology, and on the zone of the plasma. The plasma jet has been divided on two regions: hot and cold (Ref 25). When the particles are treated in the hot zone of the plasma they are accelerated, heated, and melted producing the splats. In case of nanosized particles characterized by the ability to agglomerate, with the size distribution between 0.1 and $3 \mu \mathrm{m}$ in Ref 25 , smaller particles are evaporated and bigger ones, formed due to agglomeration, explode upon solvent evaporation resulting in molten particles. In colder zone of the plasma jet Fauchais et al. have noticed that only tiny particles below $0.3 \mu \mathrm{m}$ are treated in condensation and re-solidification or evaporation and re-condensation processes. The results obtained by pulsed laminar plasma jet show the possibility of control of material injection into different zones of modulated plasma. This can allow controlling some of the thermo-physical processes occurring at the droplet scale, such as the evaporation of smaller particles in the hot zone of the plasma, shown by Fauchais et al. (Ref 25). The choice of the injection time delay can have an effect on solvent vaporization as presented in Fig. 9 and also probably on further droplet heating and coating buildup. The work on these processes and on coatings elaboration is in progress.

\section{Conclusions}

The studies of improving methods of heat and momentum transfers between plasma and material in SPPS, involving the reduction of arc instabilities using additional resonator or by means of new torches producing the plasma, significantly less fluctuated, but sill nonuniform, are presented. The examination of current methods leads to a new approach to plasma fluctuations. A special plasma torch is designed and built up, able to produce at moderate power a pulsed laminar plasma jet of nitrogen. This is obtained by phase locking Helmholtz oscillation and rearcing events in the nozzle, so that the torch works in a resonant mode, called "mosquito" mode, due to the sharp monotonic sound emitted by the torch. The plasma jet exhausts as plasma balls containing $0.5 \mathrm{~J}$ each and delivered at a frequency $1-2 \mathrm{kHz}$. The arc voltage recorded presents a very regular saw-tooth-shaped waveform and the local specific enthalpy is modulated with a ratio $h_{\max } / h_{\min }=13$, with a mean value of $13.3 \mathrm{MJ} / \mathrm{kg}$. This modulation of voltage can be used to synchronize the plasma with an appropriate injection system. The analysis of injection techniques results in selecting the system based on DOD method which allows injecting the suspension in a chosen moment. The specific features of the arc voltage signal are used to trigger an injection device that delivers calibrated suspension droplets thanks to a piezoelectric actuator. The interaction between the periodic plasma flow and the droplets is investigated optically using time-resolved imaging techniques triggered by arc voltage signal and synchronized with suspension injection. The mosquito mode generates a pulsed plasma flow that shows a very stable and spatial coherent pattern. The thermal treatment of imbedded material is shown to be very sensitive on the moment at which each droplet penetrates the plasma. This type of synchronous injection, provided the use of a periodic pulsed arc jet, presents a great interest for the control of dynamic and thermal interaction between the plasma balls and imbedded reactive species, both from an academic point of view or for application to coating elaboration, which will be investigating in a future work.

\section{Acknowledgments}

The French National Research Agency is acknowledged for financial support in the frame of PLASMAT Program (ANR-12-JCJC-JS09-0006-01).

\section{References}

1. C. Monterrubio-Badillo, H. Ageorges, T. Chartier, J.F. Coudert, and P. Fauchais, Preparation of $\mathrm{LaMnO}_{3}$ Perovskite Thin Films by Suspension Plasma Spraying for SOFC Cathodes, Surf. Coat. Technol., 2006, 200, p 3743-3756

2. F.-L. Toma, L.M. Berger, D. Jacquet, D. Wicky, I. Villaluenga, Y.R. de Miguel, and J.S. Lindelov, Comparative Study on the Photocatalytic Behaviour of Titanium Oxide Thermal Sprayed Coatings from Powders and Suspensions, Surf. Coat. Technol., 2009, 203, p 2150-2156

3. R. Henne, Solid Oxide Fuel Cells: A Challenge for Plasma Deposition Processes, J. Therm. Spray Technol., 2007, 16, p 381403

4. Z. Duan and J. Heberlein, Arc Instabilities in a Plasma Spray Torch, J. Therm. Spray Technol., 2002, 11, p 44-51

5. V. Rat and J.F. Coudert, Influence of Configuration and Operating Conditions on the Electric Arc Instabilities of a Plasma Spray Torch: Role of Acoustic Resonance, J. Phys. D Appl. Phys., 2008, 41, p 205208

6. V. Rat and J.F. Coudert, Acoustic Stabilization of Electric Arc Instabilities in Nontransferred Plasma Torches, Appl. Phys. Lett., 2010, 96, p 101503

7. M. Vilotijevic, P. Markovic, S. Zec, S. Marinkovic, and V. Jokanovic, Hydroxyapatite Coatings Prepared by a High Power Laminar Plasma Jet, J. Mater. Proc. Technol., 2011, 211, p 9961004

8. M. Vilotijevic, B. Dacic, and D. Bozic, Velocity and Texture of a Plasma Jet Created in a Plasma Torch with Fixed Minimal Arc Length, Plasma Sources Sci. Technol., 2009, 18, p 015016

\section{(n)}


9. J. Schein, J. Zierhut, M. Dzulko, G. Forster, and K.D. Landes, Improved Plasma Spray Torch Stability Through Multi-Electrode Design, Contrib. Plasma Phys., 2007, 47, p 498-504

10. J. Oberste Berghaus, B. Marple, and C. Moreau, Suspension Plasma Spraying of Nanostructured WC-12Co Coatings, $J$. Therm. Spray Technol., 2006, 15(4), p 676-681

11. P. Fauchais and A. Vardelle, Solution and Suspension Plasma Spraying of Nanostructure Coatings, Advanced Plasma Spray Applications, H.S. Jazi, Ed., InTech, Rijeka, 2012, p 149-185

12. O. Marchand, L. Girardot, M.P. Planche, P. Bertrand, Y. Bailly, and G. Bertrand, An Insight into Suspension Plasma Spray: Injection of the Suspension and Its Interaction with the Plasma Flow, J. Therm. Spray Technol., 2011, 20, p 1310-1320

13. J. Fazilleau, C. Delbos, V. Rat, J.F. Coudert, P. Fauchais, and B. Pateyron, Phenomena Involved in Suspension Plasma Spraying Part 1: Suspension Injection and Behavior, Plasma Chem. Plasma Process., 2006, 26, p 371-391

14. P. Blazdell and S. Kuroda, Plasma Spraying of Submicron Ceramic Suspensions Using a Continuous Ink Jet Printer, Surf. Coat. Technol., 2000, 123, p 239

15. V. Rat and J.F. Coudert, Pressure and Arc Voltage Coupling in dc Plasma Torches: Identification and Extraction of Oscillation Modes, J. Appl. Phys., 2010, 108, p 043304

16. J.F. Coudert and V. Rat, The Role of Torch Instabilities in the Suspension Plasma Spraying Process, Surf. Coat. Technol., 2010, 205, p 949-953

17. J. Krowka, V. Rat, and J.F. Coudert, Resonant Mode for a dc Plasma Spray Torch by Means of Pressure-Voltage Coupling:
Application to Synchronized Liquid Injection, J. Phys. D Appl. Phys., 2013, 46, p 224018

18. J. Krowka, V. Rat, and J.F. Coudert, Investigation and Control of dc Arc Jet Instabilities to Obtain a Self-Sustained Pulsed Laminar Arc Jet, J. Phys. D Appl. Phys., 2013, 46, p 505206

19. R. Noguera, M. Lejeune, and T. Chartier, 3D Fine Scale Ceramic Components Formed by Ink-Jet Prototyping Process, J. Eur. Ceram. Soc., 2005, 25, p 2055

20. S. Basu, E.H. Jordan, and B.M. Cetegen, Fluid Mechanics and Heat Transfer of Liquid Precursor Droplets Injected into HighTemperature Plasmas, Therm. Spray Technol., 2008, 17, p 60-72

21. A. Saha, S. Seal, B. Cetegen, E. Jordan, A. Ozturk, and S. Basu, Thermo-Physical Processes in Cerium Nitrate Precursor Droplets Injected into High Temperature Plasma, Surf. Coat. Technol., 2009, 203, p 2081-2091

22. M. Boulos, P. Fauchais, and E. Pfender, Thermal Plasmas, Fundamentals and Applications, Vol 1, Plenum Press, New York, 1994

23. C. Xi and E. Pfender, Heat Transfer to a Single Particle Exposed to Thermal Plasma, Plasma Chem. Plasma Process., 1982, 2, p $185-212$

24. C. Crowe, M. Sommerfield, and Y. Tsuji, Multiphase Flows with Droplets and Particles, CRC Press LLC, Boca Raton, FL, 1998

25. P. Fauchais, R. Etchart-Salas, V. Rat, J.F. Coudert, N. Caron, and K. Wittmann-Teneze, Parameters Controlling Liquid Plasma Spraying: Solutions, Sols, or Suspensions, J. Therm. Spray Technol., 2008, 17, p 31-59 